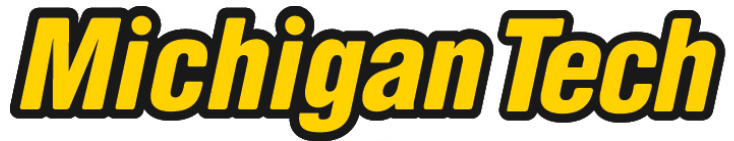 \\ Michigan Technological University Create the Future Digital Commons @ Michigan Tech
}

2014

\section{STUDY OF A DIRECT SAMPLING METHOD FOR THE INVERSE MEDIUM SCATTERING PROBLEM}

Natasha Weerasinghe

Michigan Technological University

Follow this and additional works at: https://digitalcommons.mtu.edu/etds

Part of the Mathematics Commons

Copyright 2014 Natasha Weerasinghe

\section{Recommended Citation}

Weerasinghe, Natasha, "STUDY OF A DIRECT SAMPLING METHOD FOR THE INVERSE MEDIUM SCATTERING PROBLEM", Master's report, Michigan Technological University, 2014.

https://doi.org/10.37099/mtu.dc.etds/762

Follow this and additional works at: https://digitalcommons.mtu.edu/etds

Part of the Mathematics Commons 
STUDY OF A DIRECT SAMPLING METHOD FOR THE INVERSE MEDIUM

SCATTERING PROBLEM

\title{
By
}

Nathasha Weerasinghe

\begin{abstract}
A REPORT
Submitted in partial fulfillment of the requirements for the degree of MASTER OF SCIENCE

in Mathematical Sciences
\end{abstract}

MICHIGAN TECHNOLOGICAL UNIVERSITY

2014

(c) 2014 Nathasha Weerasinghe 

This report has been approved in partial fulfillment of the requirements for the Degree of MASTER OF SCIENCE in Mathematical Sciences.

Department of Mathematical Sciences

Report Advisor: Dr. Jiguang Sun

Committee Member: Dr. Allan Struthers

Committee Member: Dr. Jingfeng Jiang

Department Chair: Dr. Mark S. Gockenbach 



\section{Contents}

List of Figures $\ldots \ldots \ldots \ldots \ldots \ldots \ldots \ldots \ldots$. . . . . . . . . . . . . . . .

Abstract $\ldots \ldots \ldots \ldots \ldots \ldots \ldots \ldots \ldots \ldots \ldots \ldots \ldots$

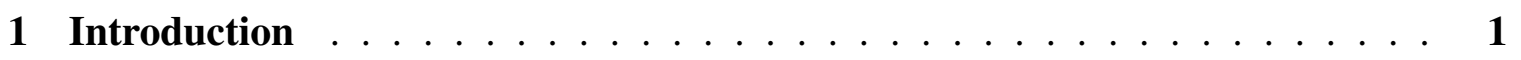

1.1 Inverse Scattering Theory $\ldots \ldots \ldots \ldots \ldots$

1.2 Methodology . . . . . . . . . . . . . . . . 3

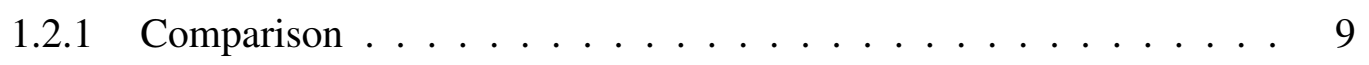

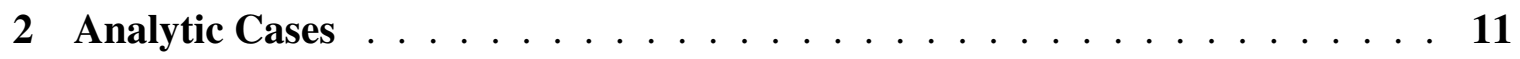

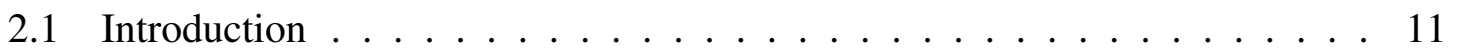

2.2 Numerical Examples $\ldots \ldots \ldots \ldots \ldots \ldots$

2.2.1 One Incident Wave $\ldots \ldots \ldots \ldots$

2.2.1.1 Different wavenumbers $\ldots \ldots \ldots \ldots$

2.2.1.2 Moving the observation curve $\ldots \ldots \ldots \ldots$

2.2.1.3 Moving the sampling domain (mesh) $\ldots \ldots \ldots$

2.2.1.4 Moving the observation curve and the sampling domain (mesh) at the same time $\ldots \ldots \ldots \ldots$ 
2.2.2 Multiple Incident Waves . . . . . . . . . . . . . 21

2.2.2.1 Observation curve is a circle . . . . . . . . . . 21

2.2.2.2 Observation curve is three quarters of a circle . . . . . 22

2.2.2.3 Observation curve is half a circle . . . . . . . . 23

2.2.2.4 Observation curve is quarter of a circle . . . . . . 23

3 General Cases $\ldots \ldots \ldots \ldots \ldots$

3.1 Numerical Examples . . . . . . . . . . . . . . . 31

4 Conclusions and Future Works . . . . . . . . . . . . . . . . . . 39

References ............................ 41 


\section{List of Figures}

$1.1 \Omega$ is the inhomogeneous medium with refractive index $q \neq 1 . \quad \ldots \ldots$

2.1 Diagram for the analytic case where the scatterer is a circular disk D. The

measurement curve $\Gamma$ is a large circle with the same center. . . . . . . . 12

2.2 Numerical results for different wavenumbers (2.2.1.1) (a) when $k=2$, (b)

when $k=5$, (c) when $k=10$, (d) when $k=50 \quad \ldots \ldots \ldots \ldots$

2.3 Numerical results for moving the observation curve $2.2 .1 .2 \ldots \ldots$

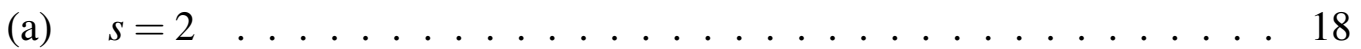

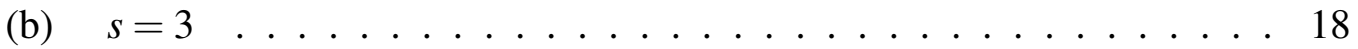

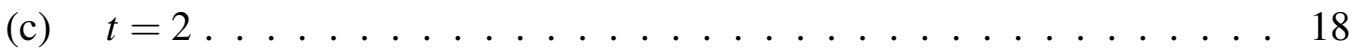

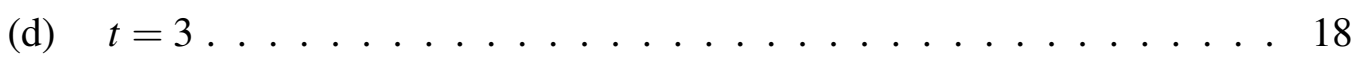

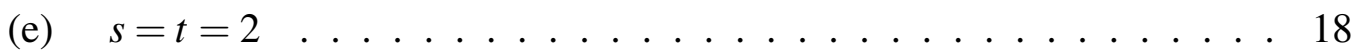

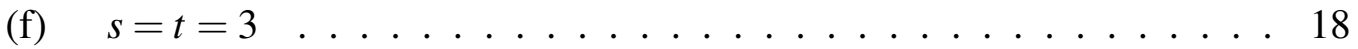

2.4 Numerical results for moving the sampling domain $2.2 .1 .3 \ldots \ldots$

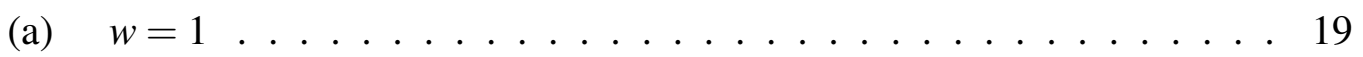

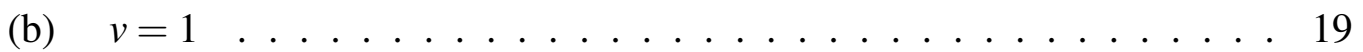

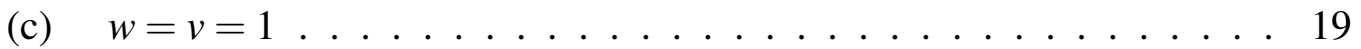


2.5 Numerical results for moving the observation curve and the sampling domain $2.2 .1 .4 \ldots \ldots \ldots \ldots$

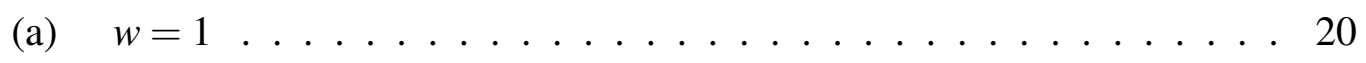

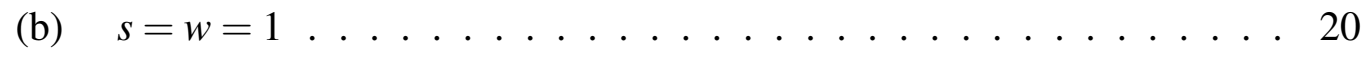

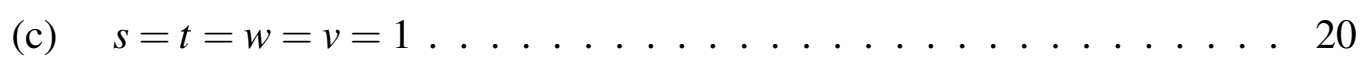

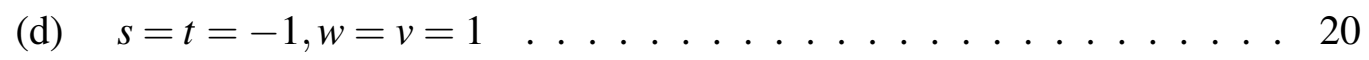

2.6 Numerical results for the analytic case when $\Gamma$ is a circle: (a) Incident wave direction, (b) when $k=5$, (c) when $k=10 \ldots \ldots \ldots$

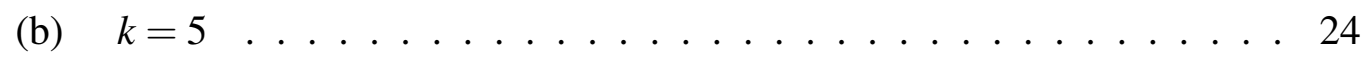

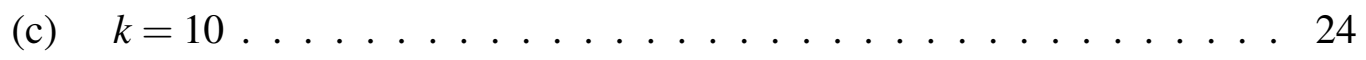

2.7 Numerical results for one incident wave when $\Gamma$ is three quarters of a circle.

Left side shows the wave directions and the right side shows the resulting

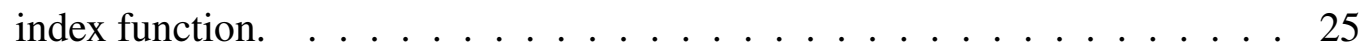

2.8 Numerical results for several incident waves when $\Gamma$ is three quarters of a circle. Left side shows the wave directions and the right side shows the resulting index function. ...........................

2.9 Numerical results for three incident waves when $\Gamma$ is three quarters of a circle. Left side shows the wave directions and the right side shows the resulting index function. . . . . . . . . . . . . 27 
2.10 Numerical results for several incident waves when $\Gamma$ is half a circle. Left side shows the wave directions and the right side shows the resulting index

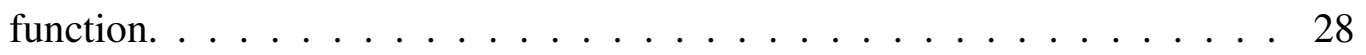

2.11 Numerical results for several incident waves when $\Gamma$ is quarter of a circle. Left side shows the wave directions and the right side shows the resulting index function. . . . . . . . . . . . . . . 29

3.1 Numerical results for example 1: (c) Reconstruction when $k=3$, (d) Reconstruction when $k=5 \ldots \ldots \ldots \ldots$. . . . . . . . . . . .

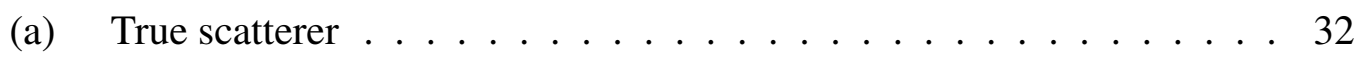

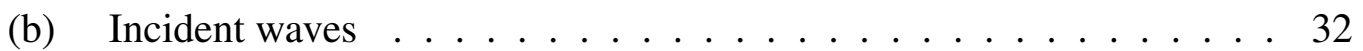

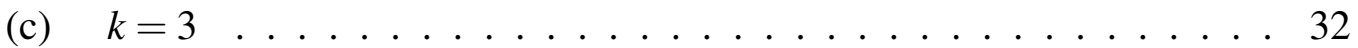

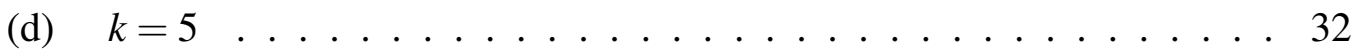

3.2 Numerical results for example 2: Reconstruction when $k=5 \ldots \ldots$

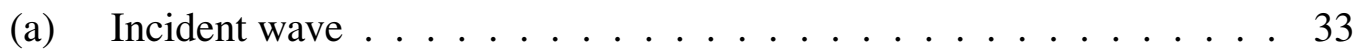

(b) True scatterer .............................

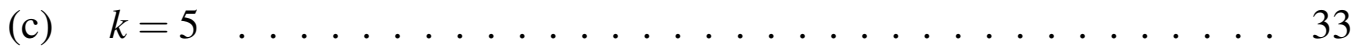

3.3 Numerical results for example 3: Left side shows the true scatterers while the right side shows the reconstructions for $k=5 \ldots \ldots \ldots$

(a) Incident wave . . . . . . . . . . . . . . 34

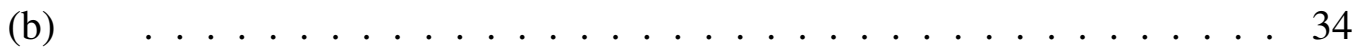

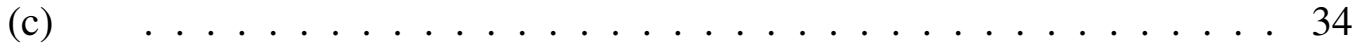


(d)

(e)

3.4 Numerical results for example 4: Reconstructions when $k=3$ and $k=5$. 35

(a) Incident wave . . . . . . . . . . . . . . . 35

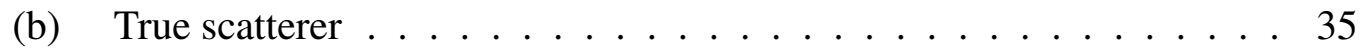

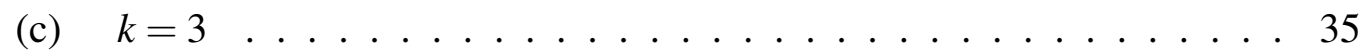

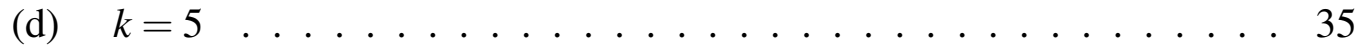

3.5 Numerical results for example 5: Reconstructions when $k=3$ and $k=5$. . 37

(a) True scatterer ...............................

(b) Incident waves . . . . . . . . . . . . . . . 37

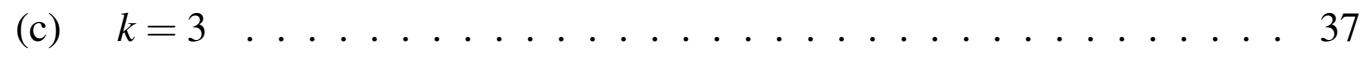

(d) $\quad k=5 \ldots \ldots \ldots \ldots$ 


\section{Abstract}

Direct sampling methods are increasingly being used to solve the inverse medium scattering problem to estimate the shape of the scattering object. A simple direct method using one incident wave and multiple measurements was proposed by Ito, Jin and Zou [1]. In this report, we performed some analytic and numerical studies of the direct sampling method. The method was found to be effective in general. However, there are a few exceptions exposed in the investigation. Analytic solutions in different situations were studied to verify the viability of the method while numerical tests were used to validate the effectiveness of the method. 


\section{Chapter 1}

\section{Introduction}

\subsection{Inverse Scattering Theory}

Obstacles or inhomogeneities have the ability to scatter the incoming wave formations. By studying these scattered waves, the characteristics of the obstacles/inhomogeneities, such as the size, the shape and the internal constitution, can be found. This is known as the inverse scattering problem (direct scattering problem is determining the scattered wave for a known object).

For example, dolphins and bats are able to figure out their surroundings using scattered waves. Acoustic location is used by these animals as well as in sonar. Medical imaging uses the scattering of ultrasound waves to get the images of the human body and inverse 
scattering problem is used in locating oil reservoirs via the reflection of seismic waves. In this report, we consider the simplest acoustic waves.

If $p=p(x, t)$ is the pressure of the fluid where the sound/acoustic waves are propagated, from the linearized Euler equation and the wave equation, we get [2]

$$
\frac{1}{c^{2}} \frac{\partial^{2} p}{\partial t^{2}}=\Delta p
$$

Here, $c=c(x)$ is the local speed of sound and fluid velocity is proportional to $\operatorname{gradp}$. For the time harmonic acoustic waves of the form

$$
p(x, t)=\operatorname{Re}\left\{u(x) e^{-i \omega t}\right\}
$$

with frequency $\omega>0$, the complex valued space dependent part satisfies the reduced wave equation

$$
\Delta u+\frac{\omega^{2}}{c^{2}} u=0
$$

The speed of sound in a homogeneous medium is a constant. Thus, by $(\sqrt{1.3})$, the Helmholtz equation is

$$
\Delta u+k^{2} u=0
$$

where $k=\omega / c$ is the wavenumber. 


\subsection{Methodology}

A direct sampling method for a time harmonic inverse medium scattering problem (IMSP) is discussed in [1]. Suppose that a bounded region $\Omega$ with a homogeneous background $\mathbb{R}^{N}(N=2,3)$ has an inhomogeneous medium inside $\Omega$. Now consider the situation where an incident plane wave is projected with a direction $d$ and a wavenumber $k$. Let the equation of the incident wave be of the form $u^{i n c}=e^{i k x . d}$ where $d$ is the direction of the plane wave. Then the total field can be considered as $u=u^{i n c}+u^{s}$ where $u^{s}$ is the scattered field due to the inhomogeneous medium. Thus, the total field induced by the inhomogeneous medium would satisfy the Helmholtz equation [2]

$$
\begin{gathered}
\Delta u+k^{2} q^{2}(x) u=0 \text { in } \mathbb{R}^{N} \\
u=u^{i}+u^{s}
\end{gathered}
$$

where $u^{i}$ is the incident field and $u^{s}$ is the scattered field induced by the inhomogeneous medium, $k$ is the wavenumber and $q(x)$ is the index of refraction 11 of the inhomogeneous medium. Moreover, $u^{s}$ needs to satisfy the Sommerfeld radiation condition as $x$ goes to infinity.

\footnotetext{
${ }^{1}$ Refractive Index - Ratio of the wave speed in the homogeneous background to that in the inhomogeneous medium at $x$
} 
Let $G(x, y)$ be the fundamental solution to the Helmholtz equation. Then

$$
G(x, y)= \begin{cases}\frac{i}{4} H_{0}^{1}(k|x-y|) & \text { if } d=2 \\ \frac{1}{4 \pi} \frac{e^{-i k|x-y|}}{|x-y|} & \text { if } d=3\end{cases}
$$

Here, $H_{0}^{1}$ is the zeroth order Hankel function of the first kind.

If we consider $q^{2}-1$, it vanishes outside the inhomogeneous medium. Let $\Omega$ be the compact support of $q^{2}-1$, i.e., $\Omega$ is the inhomogeneity. The inverse problem we are interested in is to reconstruct the shape of $\Omega$.

We can define a coefficient function $\eta(x)=\left(q^{2}(x)-1\right) k^{2}$ and then can get the induced current by the inhomogeneous medium as $I=\eta u$. Thus, the scattered field can be written as [2]

$$
u^{s}=\int_{\Omega} G(x, y) I(y) d y
$$

which makes the total field satisfy

$$
u=u^{i n c}+\int_{\Omega} G(x, y) I(y) d y
$$

From (1.9), one has that

$$
I=\eta u^{i}+\eta \int_{\Omega} G(x, y) I(y) d y
$$


The direct sampling method discussed in [1] can be used to determine the shape of

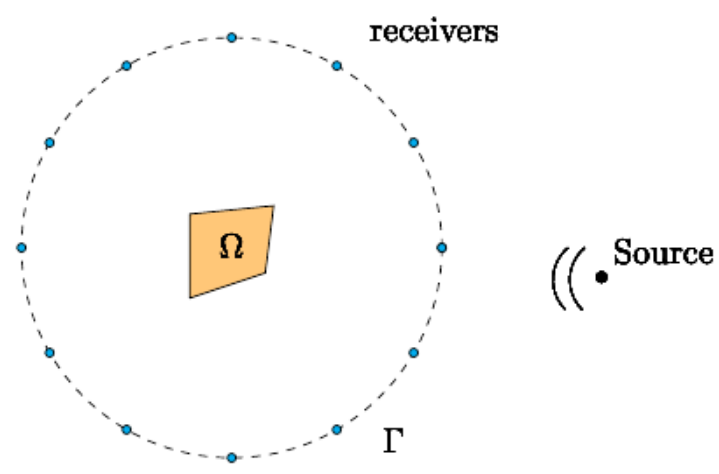

Figure 1.1: $\Omega$ is the inhomogeneous medium with refractive index $q \neq 1$.

an inhomogeneity. In the following, we give an introduction of the method. First, we consider a curve $\Gamma$ which encloses the inhomogeneous medium (see Figure 1.1). Then the fundamental solution for the open field $G\left(x, x_{p}\right)$, along with the Helmholtz equation gives

$$
\Delta G\left(x, x_{p}\right)+k^{2} G\left(x, x_{p}\right)=-\delta\left(x-x_{p}\right)
$$

where $x_{p}$ is any point in $\Omega_{\Gamma}\left(\Omega_{\Gamma}\right.$ is the domain enclosed by the circular curve $\left.\Gamma\right)$ and $\delta\left(x-x_{p}\right)$ is the Dirac delta function at $x_{p}$. Let $x_{q}$ be another point in $\Omega_{\Gamma}$. Multiplying 1.11 by the conjugate $\bar{G}\left(x, x_{q}\right)$ of the fundamental solution $G\left(x, x_{q}\right)$ :

$$
\left[\Delta G\left(x, x_{p}\right)+k^{2} G\left(x, x_{p}\right)\right] \bar{G}\left(x, x_{q}\right)=-\delta\left(x-x_{p}\right) \bar{G}\left(x, x_{q}\right)
$$


Integrating 1.12 over the domain $\Omega_{\Gamma}$

$$
\int_{\Omega_{\Gamma}}\left[\Delta G\left(x, x_{p}\right)+k^{2} G\left(x, x_{p}\right)\right] \bar{G}\left(x, x_{q}\right) d x=-\int_{\Omega_{\Gamma}} \delta\left(x-x_{p}\right) \bar{G}\left(x, x_{q}\right) d x=-\bar{G}\left(x_{p}, x_{q}\right)
$$

Similarly, if we let $x_{q} \in \Omega_{\Gamma}$ and consider 1.11 , the resultant conjugate would be

$$
\Delta \bar{G}\left(x, x_{q}\right)+k^{2} \bar{G}\left(x, x_{q}\right)=-\delta\left(x-x_{q}\right)
$$

Multiplying $(1.14)$ by $G\left(x, x_{p}\right)$ and integrating over $\Omega_{\Gamma}$, we get

$$
\int_{\Omega_{\Gamma}}\left[\Delta \bar{G}\left(x, x_{q}\right)+k^{2} \bar{G}\left(x, x_{q}\right)\right] G\left(x, x_{p}\right) d x=-\int_{\Omega_{\Gamma}} \delta\left(x-x_{q}\right) G\left(x, x_{p}\right) d x=-G\left(x_{p}, x_{q}\right) .
$$

Subtracting 1.15 from 1.13

$$
\begin{aligned}
G\left(x_{p}, x_{q}\right)-\bar{G}\left(x_{p}, x_{q}\right)= & \int_{\Omega_{\Gamma}} \Delta G\left(x, x_{p}\right) \bar{G}\left(x, x_{q}\right)+k^{2} G\left(x, x_{p}\right) \bar{G}\left(x, x_{q}\right) \\
& \left.-\Delta \bar{G}\left(x, x_{q}\right) G\left(x, x_{p}\right)-k^{2} \bar{G}\left(x, x_{q}\right) G\left(x, x_{p}\right)\right] d x \\
= & \int_{\Omega_{\Gamma}}\left[\Delta G\left(x, x_{p}\right) \bar{G}\left(x, x_{q}\right)-\Delta \bar{G}\left(x, x_{q}\right) G\left(x, x_{p}\right)\right] d x .
\end{aligned}
$$

Applying Green's second theorem $\int_{D}[u \Delta v-v \Delta u] d x=\int_{\partial D}\left[u \frac{\partial v}{\partial n}-v \frac{\partial u}{\partial n}\right] d s$ to 1.16 , we get

$$
G\left(x_{p}, x_{q}\right)-\bar{G}\left(x_{p}, x_{q}\right)=\int_{\Gamma}\left[\bar{G}\left(x, x_{q}\right) \frac{\partial G\left(x, x_{p}\right)}{\partial n}-G\left(x, x_{p}\right) \frac{\partial \bar{G}\left(x, x_{q}\right)}{\partial n}\right] d s .
$$


Sommerfeld radiation condition for Helmholtz equation states

$$
\frac{\partial G\left(x, x_{p}\right)}{\partial n}=i k G\left(x, x_{p}\right)+\text { Higher } \quad \text { Order Terms }
$$

Therefore, if we consider any point $x_{p}$ or $x_{q}$ not close to the boundary $\Gamma$,

$$
\frac{\partial G\left(x, x_{p}\right)}{\partial n} \approx i k G\left(x, x_{p}\right) \quad \text { and } \quad \frac{\partial \bar{G}\left(x, x_{q}\right)}{\partial n} \approx-i k \bar{G}\left(x, x_{q}\right) .
$$

By substituting (1.18) in the right side of (1.17), we get

$$
\begin{aligned}
\int_{\Gamma} & {\left[\bar{G}\left(x, x_{q}\right) \frac{\partial G\left(x, x_{p}\right)}{\partial n}-G\left(x, x_{p}\right) \frac{\partial \bar{G}\left(x, x_{q}\right)}{\partial n}\right] d s } \\
& \approx \int_{\Gamma}\left[i k G\left(x, x_{p}\right) \bar{G}\left(x, x_{q}\right)+i k \bar{G}\left(x, x_{q}\right) G\left(x, x_{p}\right)\right] d s \\
& =2 i k \int_{\Gamma} G\left(x, x_{p}\right) \bar{G}\left(x, x_{q}\right) d s
\end{aligned}
$$

But, for any complex number $\mathrm{z}$,

$$
z-\bar{z}=x+i y-(x-i y)=2 i y .
$$

Therefore, considering the left side of 1.17 with the above argument,

$$
G\left(x_{p}, x_{q}\right)-\bar{G}\left(x_{p}, x_{q}\right)=2 i \operatorname{Im}\left(G\left(x_{p}, x_{q}\right)\right) .
$$


(1.19) and 1.20 with 1.18 gives us the following approximation

$$
2 i k \int_{\Gamma} G\left(x, x_{p}\right) \bar{G}\left(x, x_{q}\right) d s \approx 2 i \operatorname{Im}\left(G\left(x_{p}, x_{q}\right)\right)
$$

which implies

$$
\int_{\Gamma} G\left(x, x_{p}\right) \bar{G}\left(x, x_{q}\right) d s \approx k^{-1} \operatorname{Im}\left(G\left(x_{p}, x_{q}\right)\right)
$$

Consider a sampling domain $\bar{\Omega}$ where $\Omega \subset \bar{\Omega}$. Subdividing $\bar{\Omega}$ into small elements where $\tau_{j}$ is the $j$ th element and then applying Rectangular Quadrature rule in $(1.8)$, we get

$$
u^{s}(x)=\int_{\Omega} G(x, y) I(y) d y \approx \sum_{j} w_{j} G\left(x, y_{j}\right)
$$

where $y_{j} \in \tau_{j}$ and $w_{j}$ is the weight of the $j$ th element. Here, $w_{j}=\left|\tau_{j}\right| I\left(y_{j}\right)$ where $\left|\tau_{j}\right|$ is the volume of the $j$ th element. Since there is no induced current outside $\Omega, I(y)=0$ for any $y \notin \Omega$. Hence, the summation in 1.22 is only over the elements intersecting $\Omega$. Multiplying 1.22 by $\bar{G}\left(x, x_{p}\right)$ where $x_{p} \in \bar{\Omega}$ and integrating over the boundary $\Gamma$,

$$
\int_{\Gamma} u^{s}(x) \bar{G}\left(x, x_{p}\right) d s \approx \sum_{j} w_{j} \int_{\Gamma} G\left(x, y_{j}\right) \bar{G}\left(x, x_{p}\right) d s
$$


(1.21) implies

$$
\begin{aligned}
\int_{\Gamma} u^{s}(x) \bar{G}\left(x, x_{p}\right) d s & \approx \sum_{j} w_{j} k^{-1} \operatorname{Im}\left(G\left(y_{j}, x_{p}\right)\right) \\
& =k^{-1} \sum_{j} w_{j} \operatorname{Im}\left(G\left(y_{j}, x_{p}\right)\right)
\end{aligned}
$$

which is valid if $\left\{y_{j}\right\}$ and $\left\{x_{p}\right\}$ are far apart from $\Gamma$ and each other and if $\left\{\tau_{j}\right\}$ are sufficiently refined. If $x_{p}$ and $y_{j}$ are close to each other, 1.7$)$ implies $G\left(y_{j}, x_{p}\right)$ is nearly singular and therefore, 1.23 makes the summation a very large value.

Conversely, if the two points are far apart, due to the decay property of $(1.7)$, summation in 1.23 is very small. Index function for any point $x_{p} \in \bar{\Omega}$,

$$
\Phi\left(x_{p}\right)=\frac{\left|\left\langle u^{s}(x), G\left(x, x_{p}\right)\right\rangle_{L^{2}(\Gamma)}\right|}{\left\|u^{s}(x)\right\|_{L^{2}(\Gamma)}\left\|G\left(x, x_{p}\right)\right\|_{L^{2}(\Gamma)}} .
$$

If $\left|\Phi\left(x_{p}\right)\right| \approx 1, x_{p} \in \Omega$.

If $\left|\Phi\left(x_{p}\right)\right|<<1, x_{p} \notin \Omega$.

Hence, $\Phi\left(x_{p}\right)$ serves as a characteristic function of $\Omega$.

\subsubsection{Comparison}

Compared to other sampling type techniques such as multiple signal classification (MUSIC) [3, 4, 5] and linear sampling method (LSM) [6, 7] this method is computationally 
inexpensive. This is because we only have to calculate the index function 1.24 which involves evaluation of the fundamental solution $\mathrm{G}$ (given in $(1.7 \mathrm{p})$ and it's inner product with the scattered waves and there are no matrix multiplications needed in this method. Also, the method uses only one or a few incident waves, MUSIC and LSM need full- data, i.e., a lot of incident waves. 


\section{Chapter 2}

\section{Analytic Cases}

\subsection{Introduction}

In this chapter, we consider the situation where a circular disk $\mathrm{D}$ of radius $r_{D}$ formed by an inhomogeneous medium of refractive index $q$ is surrounded by a homogeneous medium (refractive index of 1). If a point source is projected towards the circular disk from a distant $r_{S}$ where $r_{S}>r_{D}$ (see Figure 2.1), the equation of the incident wave can be written using the fundamental solution.

$$
u^{i}=\frac{i}{4} H_{0}^{1}\left(k r_{S}\right)
$$




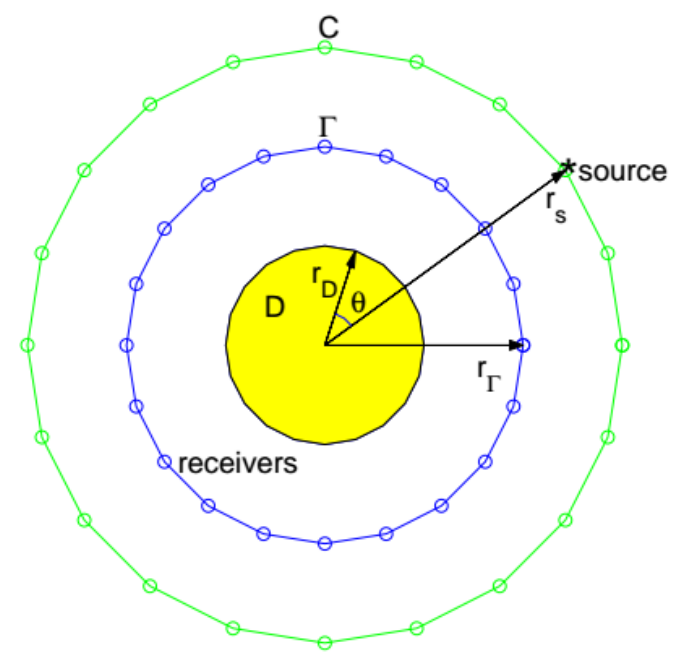

Figure 2.1: Diagram for the analytic case where the scatterer is a circular disk $\mathrm{D}$. The measurement curve $\Gamma$ is a large circle with the same center.

The Hankel function above can be expanded using the formula given below.

$$
H_{0}^{1}\left(k r_{S}\right)=J_{0}(k r) H_{0}^{1}\left(k r_{S}\right)+2 \sum_{n=1}^{\infty} J_{n}(k r) H_{n}^{1}\left(k r_{S}\right) \cos n \theta
$$

Therefore, the incident wave can be written as

$$
u^{i}(r, \theta)=\sum_{n=0}^{\infty} \gamma_{n} J_{n}(k r) \cos n \theta, \quad r \geq r_{D}
$$

where $\gamma_{0}=\frac{i}{4} H_{0}^{1}\left(k r_{S}\right) \quad$ and $\quad \gamma_{n}=\frac{i}{2} H_{n}^{1}\left(k r_{S}\right), \quad n>0$.

The scattering wave caused by the circular disk $\mathrm{D}$ of radius $r_{D}$ with an inhomogeneous 
medium, which is situated in a homogeneous medium, is given by

$$
u^{s}(r, \theta)=\sum_{n=0}^{\infty} \alpha_{n} H_{n}^{1}(k r) \cos n \theta, \quad r \geq r_{D}
$$

Here $r>r_{D}$.

Inside the scatterer, the wave form should be of the form

$$
u^{D}(r, \theta)=\sum_{n=0}^{\infty} \beta_{n} J_{n}(k q r) \cos n \theta, \quad r \geq r_{D}
$$

The transmission conditions on the boundary of the disk D (on $\partial D$ ) are

$$
\begin{array}{r}
u^{D}=u^{i}+u^{s} \\
\frac{\partial u^{D}}{\partial r}=\frac{\partial u^{i}}{\partial r}+\frac{\partial u^{s}}{\partial r}
\end{array}
$$

Substituting the equations (2.3), (2.4), and (2.5) when $r=r_{D}$ in (2.6), we get

$$
\sum_{n=0}^{\infty} \beta_{n} J_{n}\left(k q r_{D}\right) \cos n \theta=\sum_{n=0}^{\infty} \gamma_{n} J_{n}\left(k r_{D}\right) \cos n \theta+\sum_{n=0}^{\infty} \alpha_{n} H_{n}^{1}\left(k r_{D}\right) \cos n \theta
$$

which leads to

$$
\beta_{n} J_{n}\left(k q r_{D}\right)=\gamma_{n} J_{n}\left(k r_{D}\right)+\alpha_{n} H_{n}^{1}\left(k r_{D}\right)
$$


The partial derivatives of $u^{i}, u^{s}$ and $u^{D}$ are

$$
\begin{aligned}
& \frac{\partial u^{i}}{\partial r}=\sum_{n=0}^{\infty} \gamma_{n} k\left(\frac{n}{k r} J_{n}(k r)-J_{n+1}(k r)\right) \cos n \theta, \quad r \geq r_{D} \\
& \frac{\partial u^{s}}{\partial r}=\sum_{n=0}^{\infty} \alpha_{n} k\left(\frac{n}{k r} H_{n}^{1}(k r)-H_{n+1}^{1}(k r)\right) \cos n \theta, \quad r \geq r_{D} \\
& \frac{\partial u^{D}}{\partial r}=\sum_{n=0}^{\infty} \beta_{n} k q\left(\frac{n}{k q r} J_{n}(k q r)-J_{n+1}(k q r)\right) \cos n \theta, \quad r \leq r_{D}
\end{aligned}
$$

When we consider the situation $r=r_{D}$ with the transmission condition given in (2.7), we get

$$
\begin{aligned}
\beta_{n} q\left(\frac{n}{k q r_{D}} J_{n}\left(k q r_{D}\right)-J_{n+1}\left(k q r_{D}\right)\right)= & \gamma_{n}\left(\frac{n}{k r_{D}} J_{n}\left(k r_{D}\right)-J_{n+1}\left(k r_{D}\right)\right)+ \\
& \alpha_{n}\left(\frac{n}{k r_{D}} H_{n}^{1}\left(k r_{D}\right)-H_{n+1}^{1}\left(k r_{D}\right)\right)
\end{aligned}
$$

which implies

$$
\begin{aligned}
\frac{\beta_{n} n}{k r_{D}} J_{n}\left(k q r_{D}\right)-\beta_{n} q J_{n+1}\left(k q r_{D}\right)= & \frac{\gamma_{n} n}{k r_{D}} J_{n}\left(k r_{D}\right)-\gamma_{n} J_{n+1}\left(k r_{D}\right)+ \\
& \frac{\alpha_{n} n}{k r_{D}} H_{n}^{1}\left(k r_{D}\right)-\alpha_{n} H_{n+1}^{1}\left(k r_{D}\right)
\end{aligned}
$$


Thus, by equating the coefficients, we have that

$$
\begin{gathered}
\frac{\beta_{n} n}{k r_{D}} J_{n}\left(k q r_{D}\right)=\frac{\gamma_{n} n}{k r_{D}} J_{n}\left(k r_{D}\right)+\frac{\alpha_{n} n}{k r_{D}} H_{n}^{1}\left(k r_{D}\right) \Longrightarrow \beta_{n} J_{n}\left(k q r_{D}\right)=\gamma_{n} J_{n}\left(k r_{D}\right)+\alpha_{n} H_{n}^{1}\left(k r_{D}\right) \\
\beta_{n} q J_{n+1}\left(k q r_{D}\right)=\gamma_{n} J_{n+1}\left(k r_{D}\right)+\alpha_{n} H_{n+1}^{1}\left(k r_{D}\right)
\end{gathered}
$$

Multiplying (2.12) by $q J_{n+1}\left(k q r_{D}\right)$ and 2.13) by $J_{n}\left(k q r_{D}\right)$ and solving for $\alpha_{n}$, we get

$$
\alpha_{n}=\frac{J_{n}\left(k q r_{D}\right) J_{n+1}\left(k r_{D}\right)-q J_{n}\left(k r_{D}\right) J_{n+1}\left(k q r_{D}\right)}{q H_{n}^{1}\left(k r_{D}\right) J_{n+1}\left(k q r_{D}\right)-H_{n+1}^{1}\left(k r_{D}\right) J_{n}\left(k q r_{D}\right)} \gamma_{n}
$$

Now, this $\alpha_{n}$ can be used in 2.4 and the scattered wave is obtained.

\subsection{Numerical Examples}

Let's consider the analytic case in different situations. For the numerical implementations, we can consider the number of terms used to calculate the scattered wave to be large enough for the given wavenumber. When the wavenumber increases the number of terms considered needs to be increased. 


\subsubsection{One Incident Wave}

\subsubsection{Different wavenumbers}

Here the inhomogeneous object is a circular disk centered at the origin with radius $r_{D}=$ 0.02 and refractive index $q=2$. An incident wave of wavenumber $k$ is projected from a point source situated at $(6,0)$ and the scattered field $u^{s}$ was measured (by using the simulated equation) at 40 equidistant points on circle $\Gamma$ of radius 4 . The sampling domain $\bar{\Omega}$ that was considered is the square mesh $[-2,2]^{2}$ with the squares in the mesh having the equal width $m=0.04$. The numerical results for the index function $\Phi$ for different values of $k(k=2,5,10,50)$ are shown in Figure 2.2. We can see that the points on the sampling domain which are closer to the inhomogeneous object, have larger $\Phi$ values (values close to 1) whereas the points further away from the object would have $\Phi$ values close to zero. Thus, the image we get is an accurate indicator for the location of the object. But, as the wavenumber increases, we have to consider more terms when calculating the scattered wave (we used only 40 terms here). 


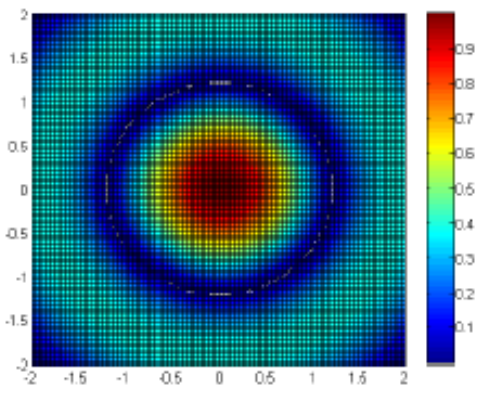

(a) $k=2$

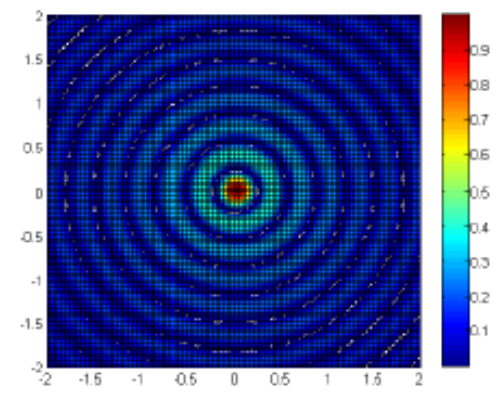

(c) $k=10$

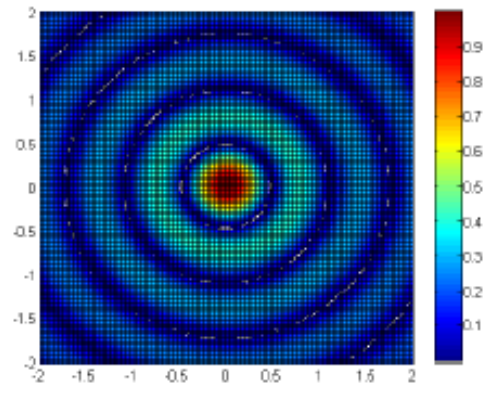

(b) $k=5$

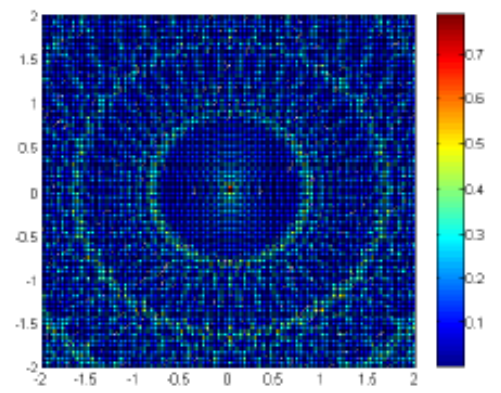

(d) $k=50$

Figure 2.2: Numerical results for different wavenumbers 2.2.1.1 (a) when $k=2$, (b) when $k=5$, (c) when $k=10$, (d) when $k=50$

\subsubsection{Moving the observation curve}

Here, we consider the same situation as explained above(in Case 1) with the wavenumber $k=5$ and try to move the observation curve $\Gamma$ without moving anything else. $s$ represents the movement of $\Gamma$ towards the negative $y$ direction and $t$ represents the movement towards the negative $x$ direction. The numerical results for the index function $\Phi$ for different movements of $\Gamma$ are shown in Figure 2.3. The position of the inhomogeneous object is clearly visible as long as the object is away from $\Gamma$. When the distance between the object 


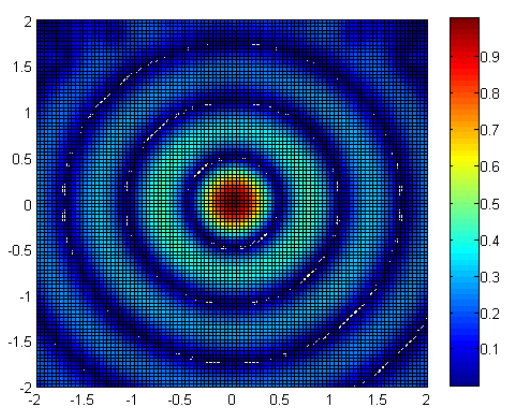

(a) $s=2$

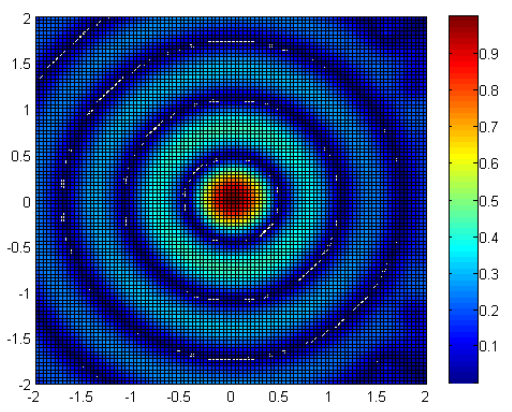

(c) $t=2$

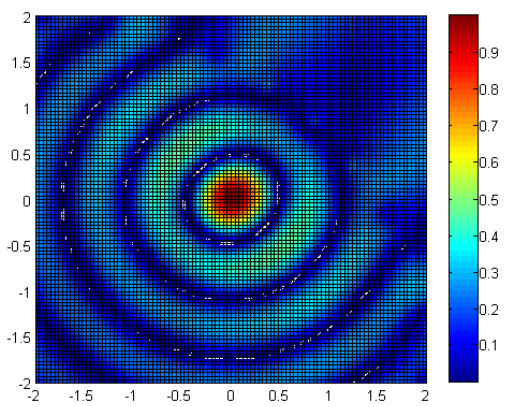

(e) $s=t=2$

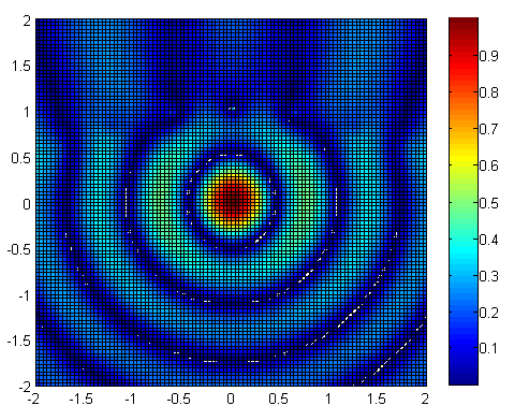

(b) $s=3$

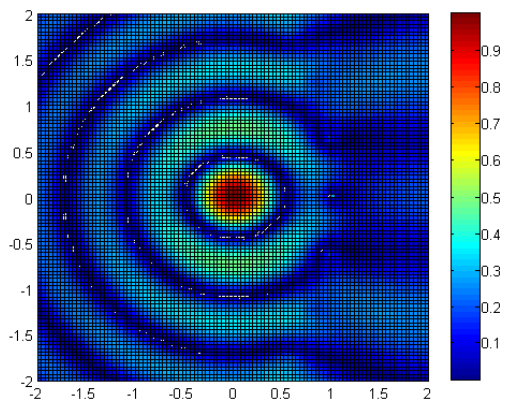

(d) $t=3$

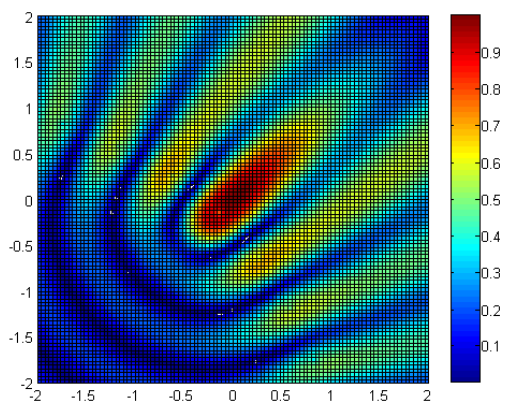

(f) $s=t=3$

Figure 2.3: Numerical results for moving the observation curve 2.2.1.2

and $\Gamma$ decreases, the image fails to give an accurate shape and position of the object. This complies with the theory behind the function $\Phi$. 


\subsubsection{Moving the sampling domain (mesh)}

Consider the original situation explained in 2.2.1.1 and then try to move only the sampling domain $\bar{\Omega}$ which is a mesh in our case. $w$ represents the movement of the mesh towards the negative $x$ direction and $v$ represents the movement towards the negative $y$ direction. The numerical results for the index function $\Phi$ for different movements of the mesh are shown in Figure 2.4. It is observed that the position of the object does not change no matter where the mesh moves.

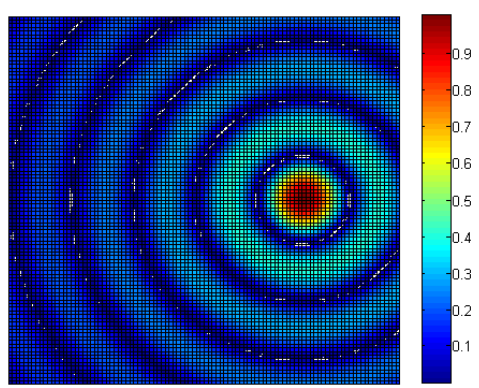

(a) $w=1$

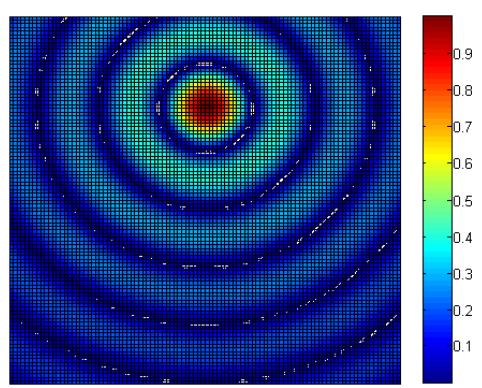

(b) $v=1$

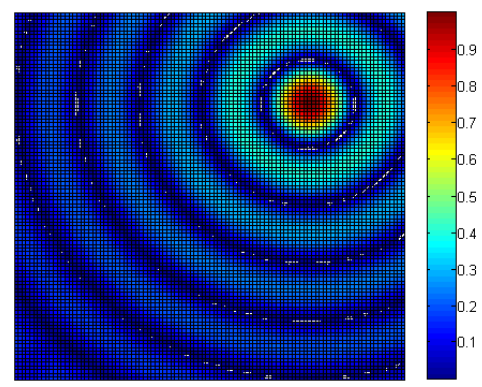

(c) $w=v=1$

Figure 2.4: Numerical results for moving the sampling domain 2.2 .1 .3 
2.2.1.4 Moving the observation curve and the sampling domain (mesh) at the same time

Here, we try to consider 2.2.1.2 and 2.2.1.3 acting together. The numerical results for several situations are given in Figure 2.5. As long as the inhomogeneous object and the observation curve $\Gamma$ have a considerate amount of distance between each other, the movement of $\Gamma$ wouldn't affect the outcome as expected.

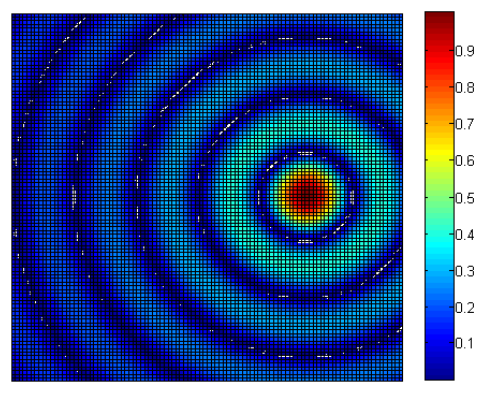

(a) $w=1$

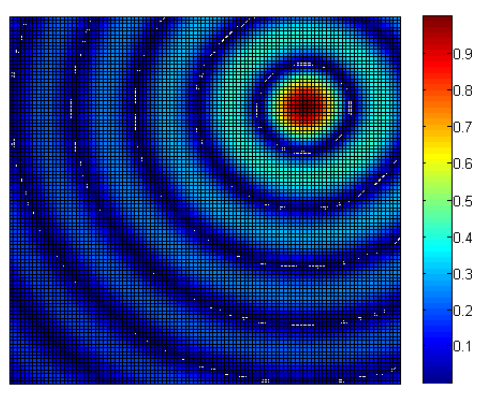

(c) $s=t=w=v=1$

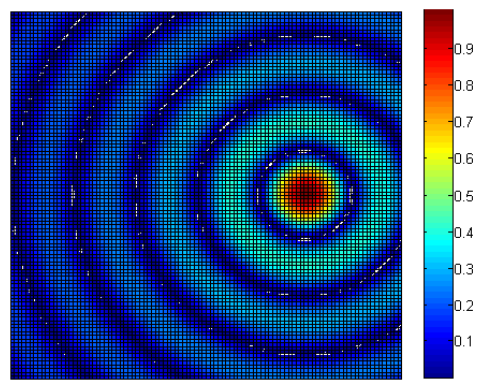

(b) $s=w=1$

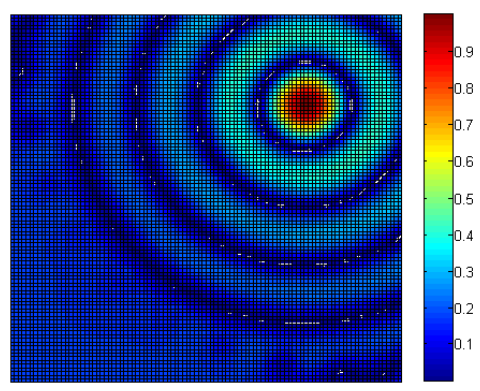

(d) $s=t=-1, w=v=1$

Figure 2.5: Numerical results for moving the observation curve and the sampling domain 2.2.1.4 


\subsubsection{Multiple Incident Waves}

In this section we consider the effect of the number of incident waves and the shape of $\Gamma$ used to detect the scattered wave. The inhomogeneous object used in this section is a circular disk centered at the origin with radius $r_{D}=0.2$ and refractive index $q=2$. Incident waves of wavenumber $k$ are projected from point sources situated 5 units away from the origin.

\subsubsection{Observation curve is a circle}

Here, we consider a circle of radius 4 as the observation curve $\Gamma$ with one, two or three incident waves in different locations.

The numerical results for the index function $\Phi$ for different positions of the incident waves are shown in Figure 2.6. When the wavenumber is 5, it can be observed that the accuracy of the result is sufficient enough no matter what the number of incident waves and their directions would be. But, when the wavenumber is higher we see that the accuracy can be improved by increasing the number of incident waves (even though increasing the number of terms used to stimulate the scattered wave is an option for the analytic case). 


\subsubsection{Observation curve is three quarters of a circle}

Case 1: Observation curve $\Gamma$ is three quarters of a circle and one incident wave

Here, we consider a sector $(-\pi / 2 \leq \theta \leq \pi)$ of a circle of radius 4 as $\Gamma$ with one incident wave in different locations. The numerical results for the index function $\Phi$ for different positions of the incident wave are shown in Figure 2.7

Case 2: $\Gamma$ is three quarters of a circle and two incident waves

Here, we consider a sector $(-\pi / 2 \leq \theta \leq \pi)$ of a circle of radius 4 as $\Gamma$ with two incident waves in different locations. The numerical results for the index function $\Phi$ for different positions of two incident waves are shown in Figure 2.8 .

Case 3: $\Gamma$ is three quarters of a circle and three incident waves

Here, we consider a sector $(-\pi / 2 \leq \theta \leq \pi)$ of a circle of radius 4 as $\Gamma$ with three incident waves in different locations and the numerical results for the index function $\Phi$ for different positions of three incident waves are shown in Figure 2.9.

Observing the cases 1, 2, and 3 in Figure 2.7, Figure 2.8, and Figure 2.9 we see that even though the imperfections around the object has increased a little bit, the shape and the size of the object is accurate for one or more incident waves in different directions. Thus, the number of incident waves or the direction would not make a difference. 


\subsubsection{Observation curve is half a circle}

Here, we consider a sector $(-\pi / 2 \leq \theta \leq \pi / 2)$ of a circle of radius 4 as the observation curve $\Gamma$ with one, two, or three incident waves in different locations. The numerical results for the index function $\Phi$ for different positions of incident waves are shown in Figure 2.10. Compared to the first and second cases, more imperfections are observed while the shape of the object is slightly deformed. Here also, the number of incident waves or the directions they are projected did not make any difference in increasing the accuracy.

\subsubsection{Observation curve is quarter of a circle}

Here, we consider a sector $(-\pi / 2 \leq \theta \leq 0)$ of a circle of radius 4 as the observation curve $\Gamma$ with one, two, or three incident waves in different locations. The numerical results for the index function $\Phi$ for different positions of incident waves are shown in Figure 2.11. It can be observed that considering quarter of a circle as $\Gamma$ (with 10 equidistanced detectors) did not contributed towards figuring out the shape, size or the position of the object. Just like in previous cases, the number of incident waves nor the directions made the result more accurate. 

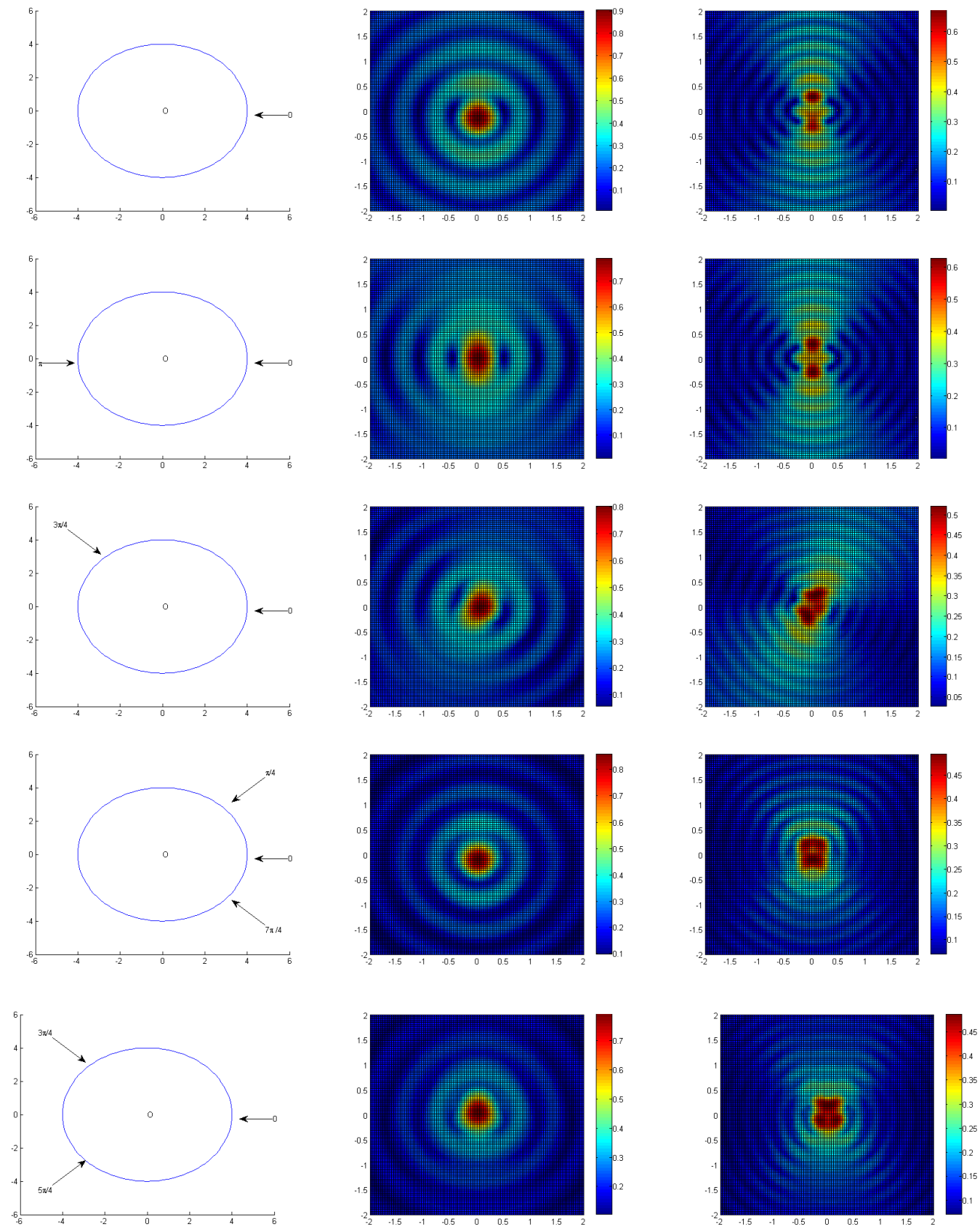

(a)

(b) $k=5$

(c) $k=10$

Figure 2.6: Numerical results for the analytic case when $\Gamma$ is a circle: (a) Incident wave direction, (b) when $k=5$, (c) when $k=10$ 

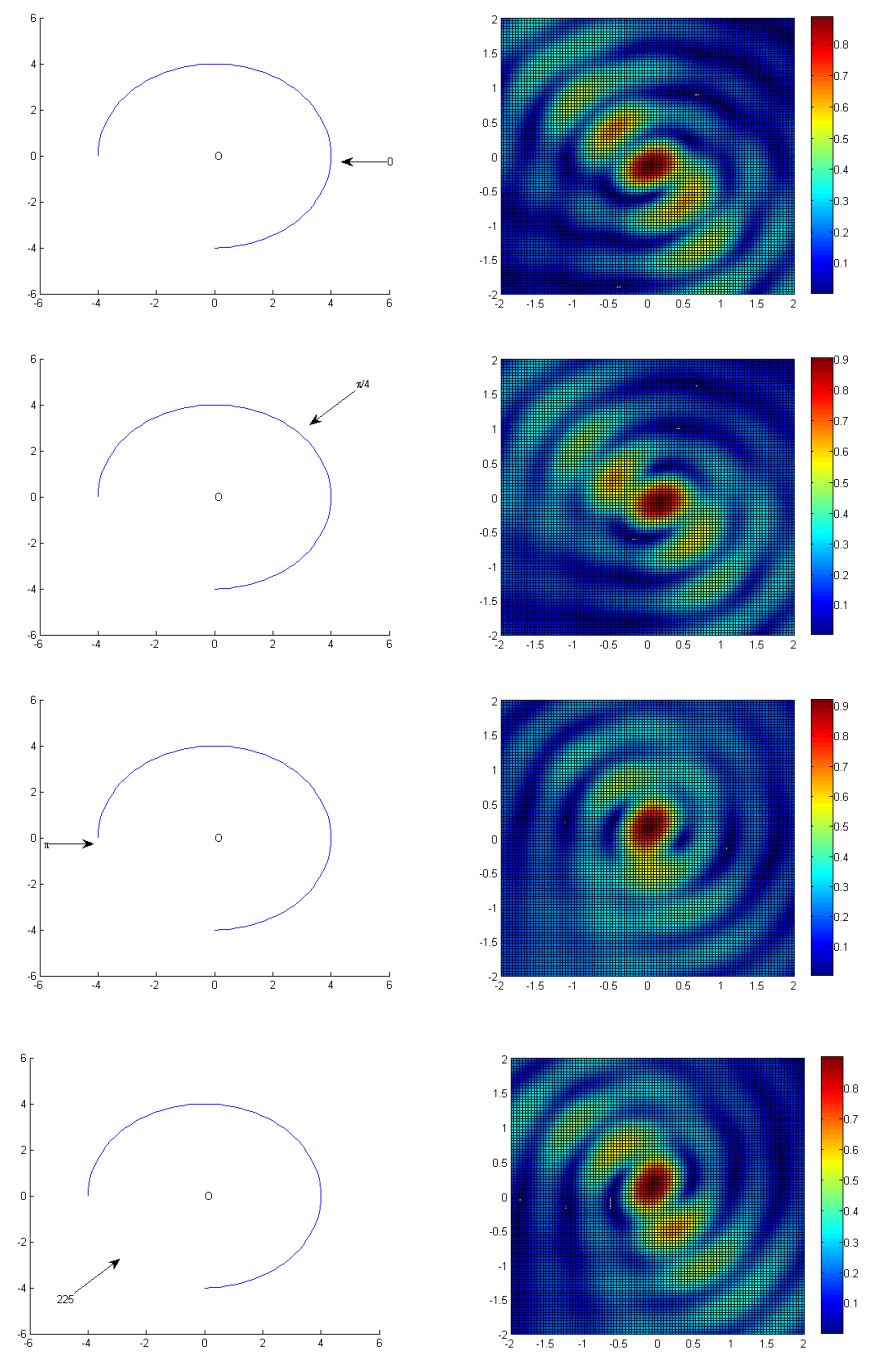

Figure 2.7: Numerical results for one incident wave when $\Gamma$ is three quarters of a circle. Left side shows the wave directions and the right side shows the resulting index function. 

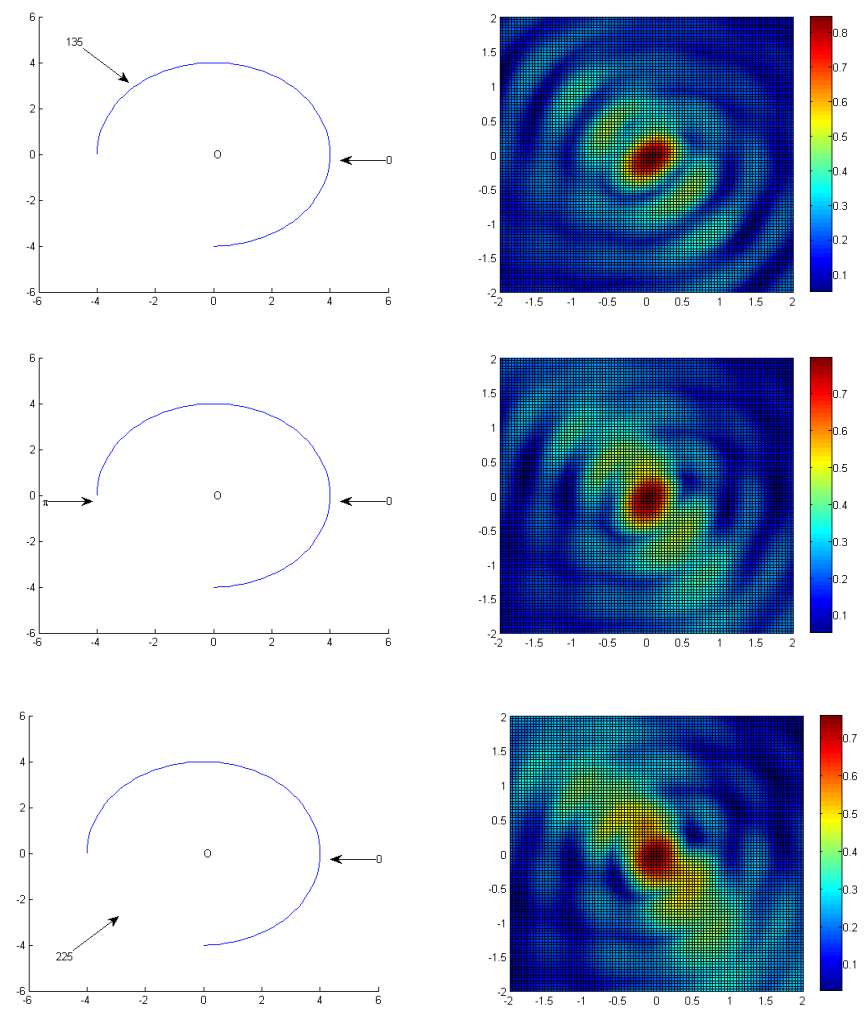

Figure 2.8: Numerical results for several incident waves when $\Gamma$ is three quarters of a circle. Left side shows the wave directions and the right side shows the resulting index function. 

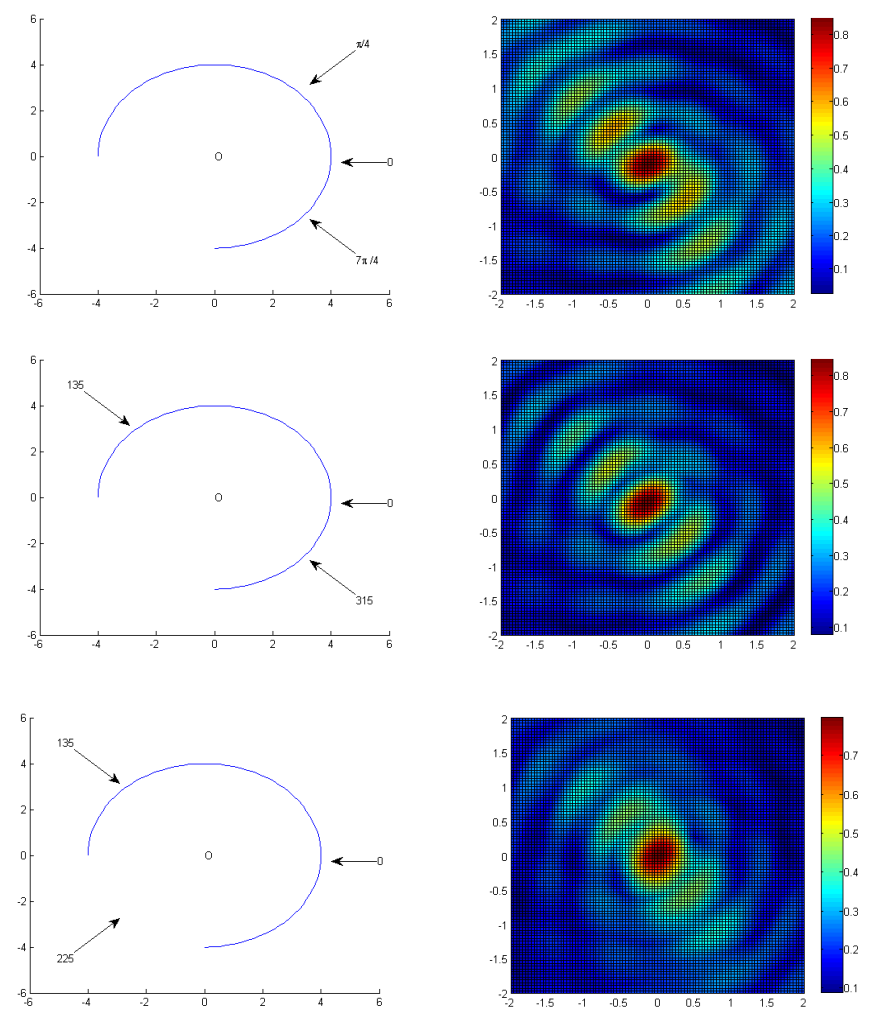

Figure 2.9: Numerical results for three incident waves when $\Gamma$ is three quarters of a circle. Left side shows the wave directions and the right side shows the resulting index function. 

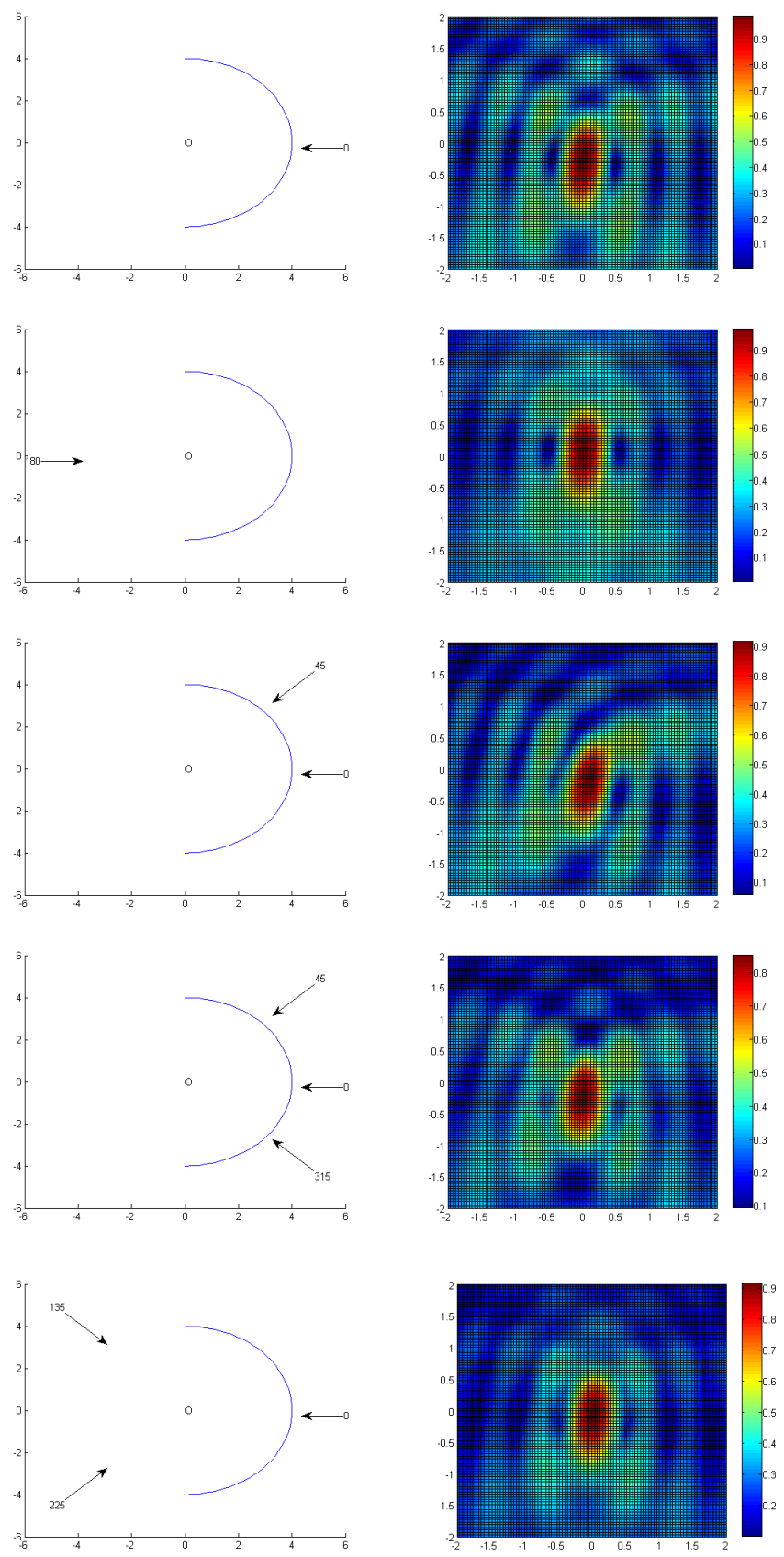

Figure 2.10: Numerical results for several incident waves when $\Gamma$ is half a circle. Left side shows the wave directions and the right side shows the resulting index function. 

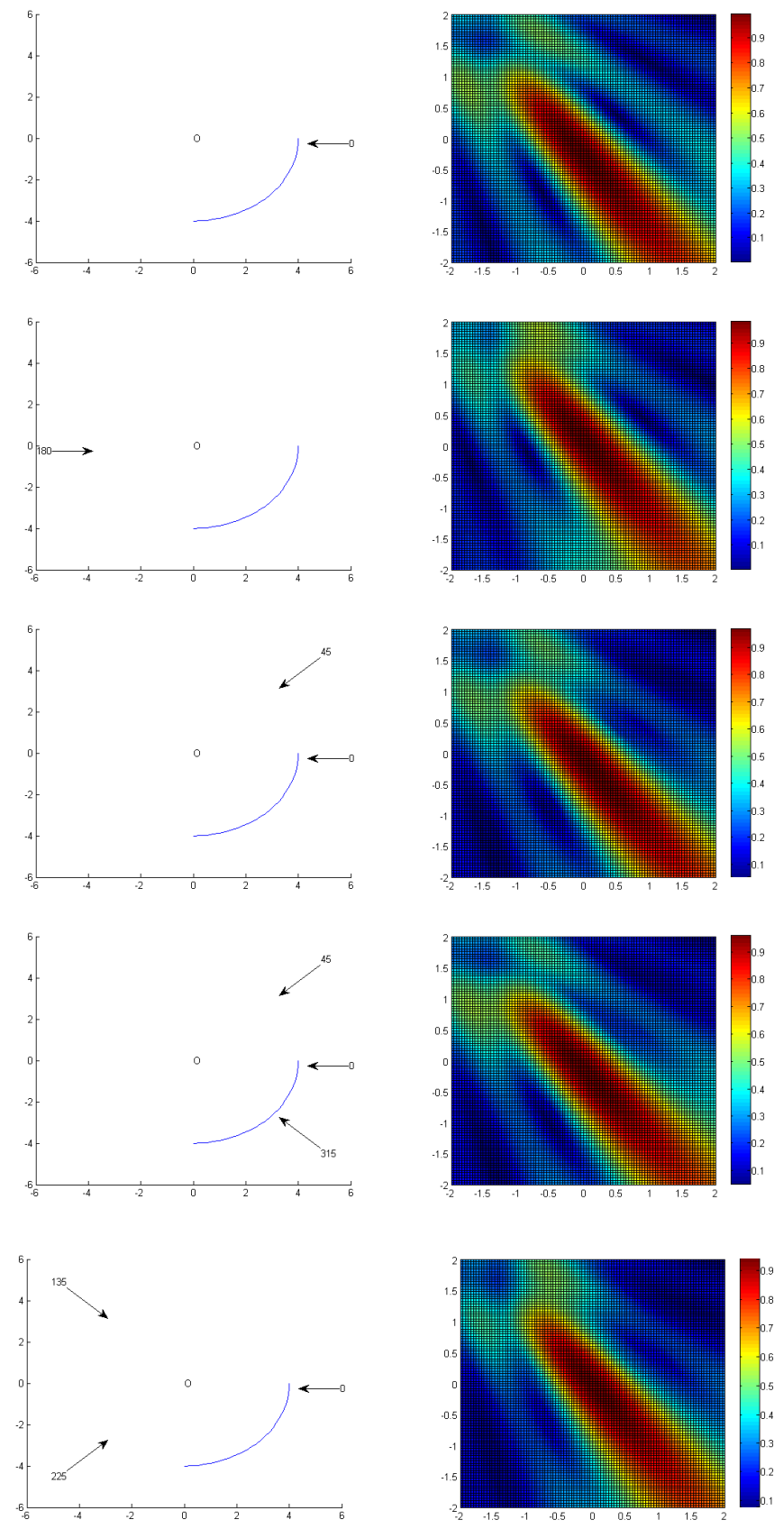

Figure 2.11: Numerical results for several incident waves when $\Gamma$ is quarter of a circle. Left side shows the wave directions and the right side shows the resulting index function. 



\section{Chapter 3}

\section{General Cases}

\subsection{Numerical Examples}

In this chapter, we'll consider several sets of scattered wave data taken from some unknown objects and try to figure out the shape, size, and the position of the object/objects using the index function (1.24). The scattered fields are computed by a finite element method. In all the examples given below, the 20 equidistanced receivers are positioned 3 units away from the origin. i.e., the $\Gamma$ considered is a circle of radius 3 .

\section{Example 1}

Here, the true scatterer is a circle of radius 0.3 with the center located at the origin. The sources are positioned at $(0,6)$ and $(6,0)$. First, only one incident wave was considered for 
wavenumbers $k=3$ and $k=5$. Next, the same procedure was done for two incident waves.

The numerical results for the index function $\Phi$ for the above four situations are shown in

Figure 3.1 .

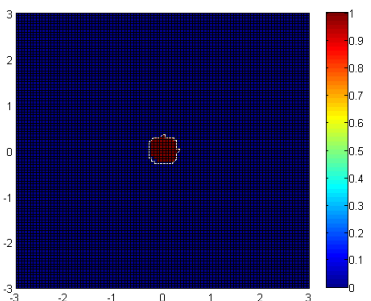

(a) True scatterer
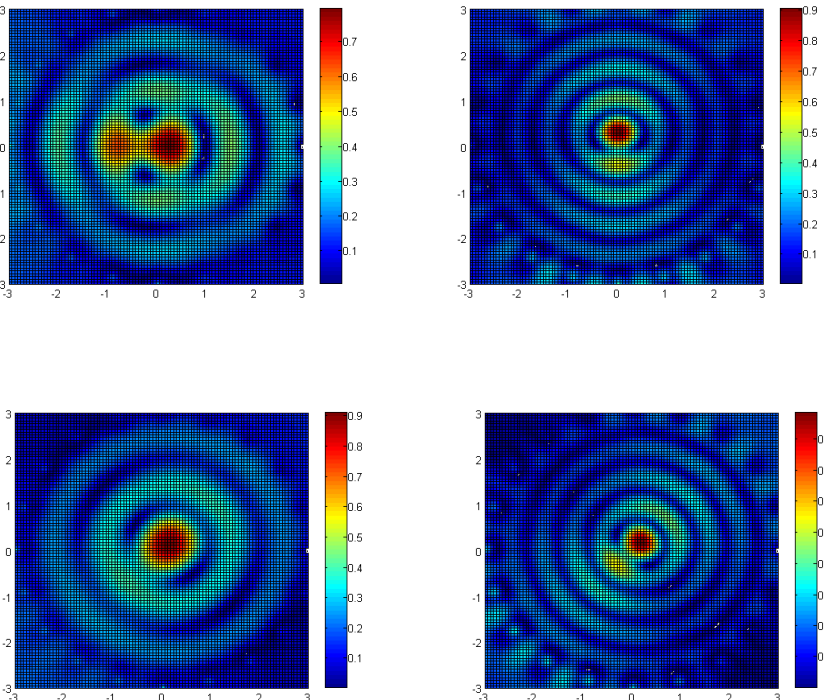

(c) $k=3$

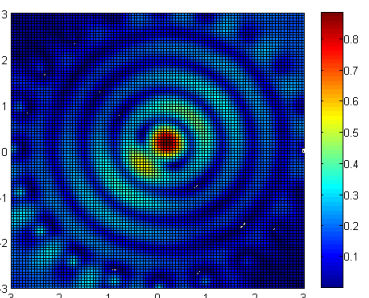

(d) $k=5$

Figure 3.1: Numerical results for example 1: (c) Reconstruction when $k=$ 3, (d) Reconstruction when $k=5$

The results obtained with one incident wave is almost as same as the results obtained with two incident waves. It can be observed that the size and the shape of the reconstructed 
scatterers are an accurate representation of the true scatterer for both the wavenumbers considered. However, the position of the scatterer has moved 0.3 points (the radius of the scatterer) towards the direction where the incident waves are projected.

\section{Example 2}

True scatterer is a circle of radius 0.3 with the center located at the point $(0.5,0.5)$. Only one incident wave with wavenumber, $k=5$ was used. The numerical result for the index function $\Phi$ is shown in Figure 3.2 .

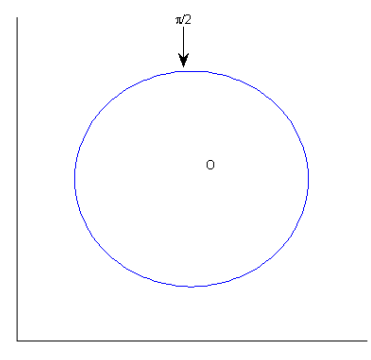

(a) Incident wave

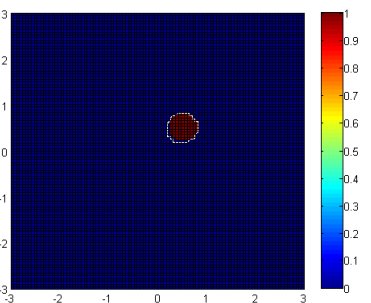

(b) True scatterer

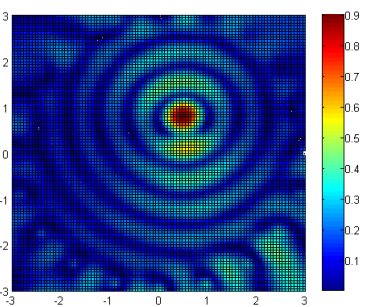

(c) $k=5$

Figure 3.2: Numerical results for example 2: Reconstruction when $k=5$

The reconstructed scatterer is an accurate representation of the true circular scatterer with respect to the shape and the size even though only one incident wave was used. But as in the previous example, the position of the scatterer has moved towards the source direction in an equal amount.

\section{Example 3}

The true scatterer in this example is a square centered at the origin. Two different sized squares were tested using one incident wave with wavenumber $k=5$. One has a side 
length of 0.25 while the other one is four times larger (side length of 1.0). The source position here is $(6,0)$. Figure 3.3 shows the numerical results for the index function.

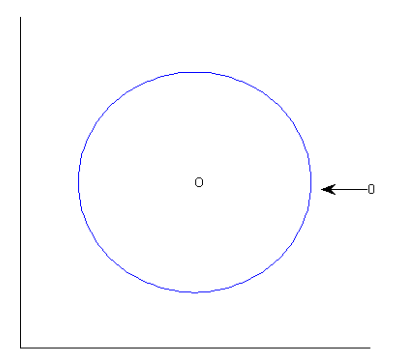

(a) Incident wave

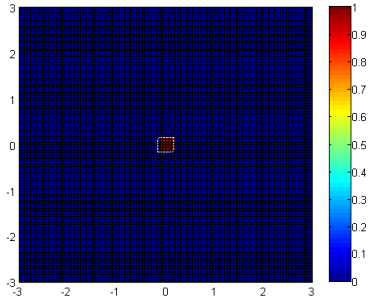

(b)

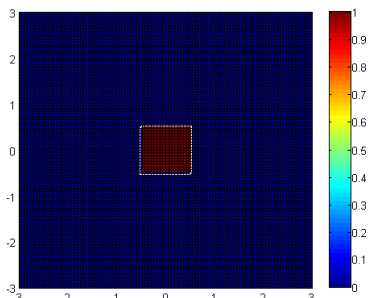

(d)

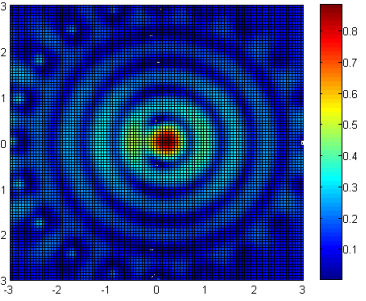

(c)

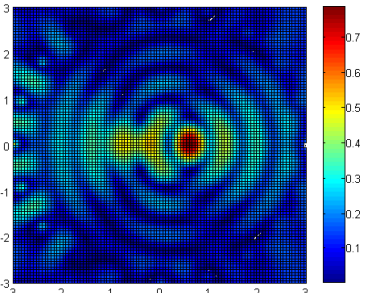

(e)

Figure 3.3: Numerical results for example 3: Left side shows the true scatterers while the right side shows the reconstructions for $k=5$

The reconstruction of the smaller of the squares agrees with the size of the true scatterer while the larger one does not. Distinguishment between the smaller square and a circle of the same size wouldn't be possible without using more than one incident wave. 


\section{Example 4}

Two square scatterers of side length 0.25 centered on $(0.5,0.5)$ and $(-0.5,-0.5)$ are tested with one incident wave where the source is positioned at $(6,0)$. Numerical results for the index function $\Phi$ are shown in Figure 3.4

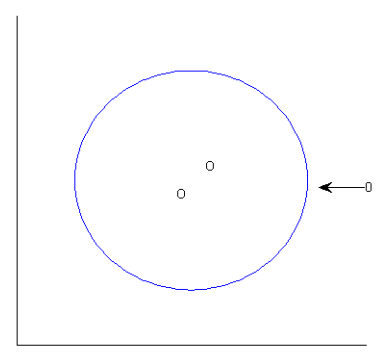

(a) Incident wave

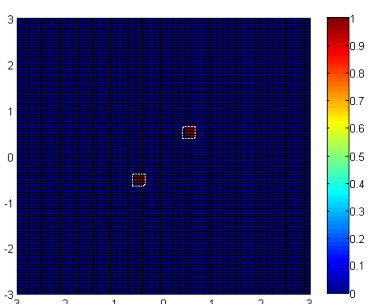

(b) True scatterer

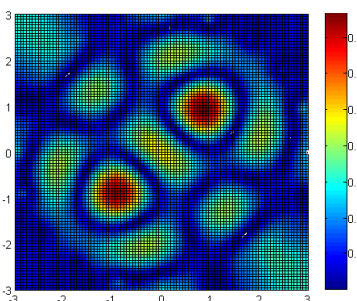

(c) $k=3$

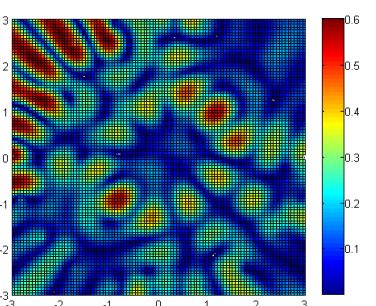

(d) $k=5$

Figure 3.4: Numerical results for example 4: Reconstructions when $k=3$ and $k=5$

Even though the reconstruction with the wavenumber $k=5$ gives a more approximated size when compared with the true scatterer, many of the other spots also show up (Figure 3.4 (d)), which is misleading. When the wavenumber equals 3 , we get a more accurate idea of the number of scatterers involved and the position of those (Figure 3.4(c)). 


\section{Example 5}

A triangular scatterer with the corner points $(-0.3,0),(-0.1,-0.5)$, and $(-0.5,-0.5)$ and a square scatterer with side length 0.5 centered at $(0.5,0.5)$ are considered with one incident wave and two incident waves respectively. The source positions are $(6,0)$ and $(0,6)$ with the wavenumbers $k=3$ and $k=5$. The numerical results for the index function are given in Figure 3.5 .

It can be observed that two incident waves give a more accurate reconstruction when compared with the true scatterer. However, even with one incident wave, we can figure out the number of scatterers and their positions. Also, the smaller wavenumber makes the image look larger than it is.

The imperfections that can be observed may be due to the oscillating behavior of the fundamental solution and the ill-posed nature of the inverse medium scattering problem. Also, the index function is an estimation of the scatterer rather than finding the exact solution (i.e., the argument considered when relating the index function with the scatterer is probabilistic and not exact). So, we can use this estimation as an initial guess for more expensive but more accurate methods like the regularized least-squares, Gauss-Newton method or contrast source inversion $[8,9,10]$. 


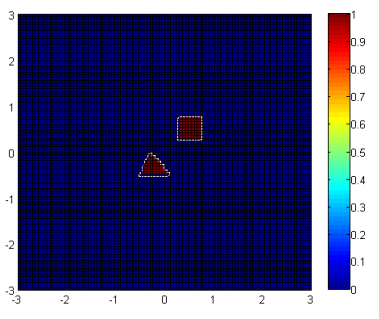

(a) True scatterer
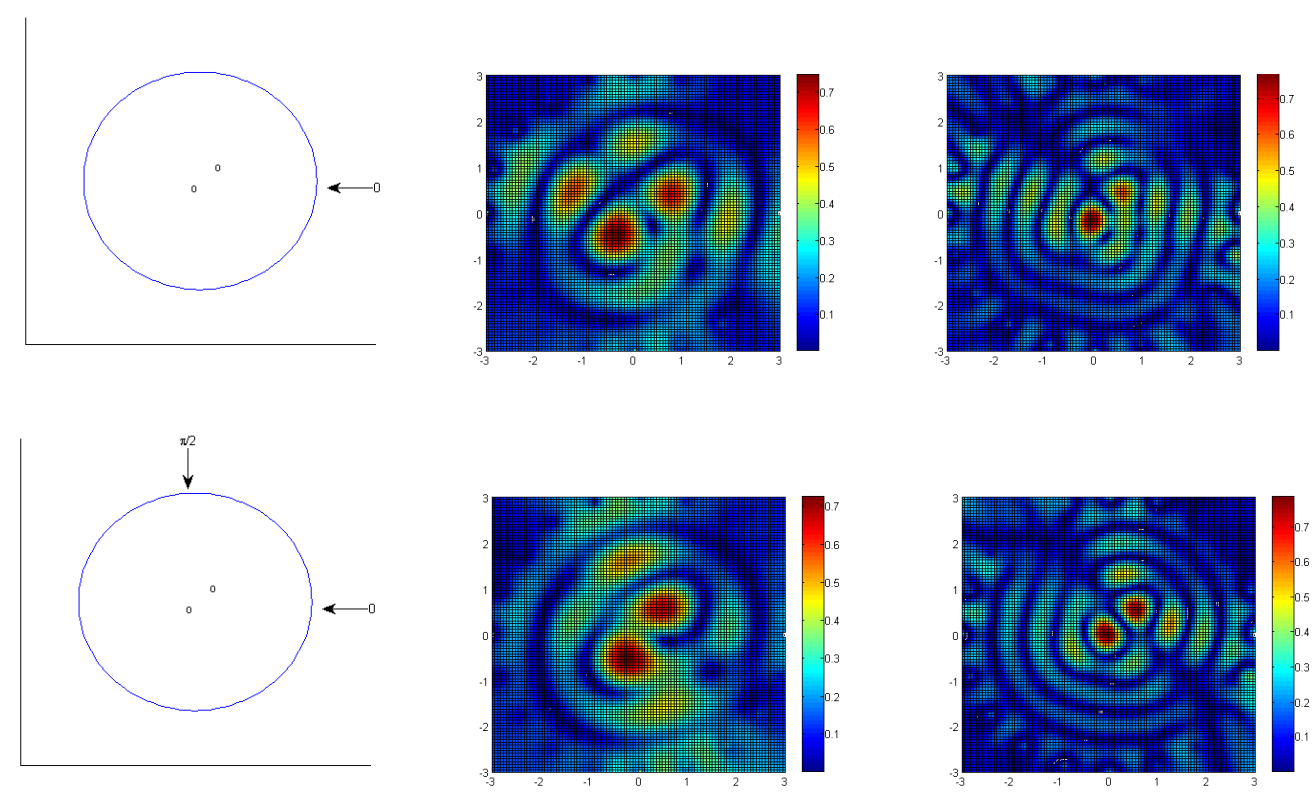

(b) Incident waves

(c) $k=3$

(d) $k=5$

Figure 3.5: Numerical results for example 5: Reconstructions when $k=3$ and $k=5$ 



\section{Chapter 4}

\section{Conclusions and Future Works}

The direct sampling method discussed in [1] works well when it's applied to the analytic case discussed in Chapter 2, if we know the correct wavenumber to use. Larger or smaller wavenumbers would not give the right idea of the size of the scatterer. The wavenumber suitable is inversely proportional to the size of the scatterer.

Also, the receivers shouldn't be too close to the scatterer which agrees with the index function $\Phi$ in $(1.24)$. Furthermore, the method works best when $\Gamma$ is a circle enclosing the scatterer and it does not work at all if the receivers cover only a quarter of the closed measurement curve. If the receivers does not enclose at least three quarters of the scatterer, the method wouldn't provide a descent outcome.

One or two incident waves would give an accurate description about the scatterer if the appropriate wavenumber is used. If the wavenumber is a bit higher, more incident waves 
would have to be used to get the same accuracy as the lower wavenumber.

The situations considered here are the cases where the inhomogeneous scatterers can be enclosed using several receivers $(\Gamma)$. Future work can be done to extend the method so that it can estimate the size, shape and the positioning of other types of scatterers. 


\section{References}

[1] K. Ito, B. Jin, and J. Zou, "A direct sampling method to an inverse medium scatteing problem," Inverse Problems, 28, 025003(11pp), 2012.

[2] D. Colton and R. Kress, Inverse Acoustic and Electromagnetic Scattering Theory. Berlin: Springer, 2nd ed., 1998.

[3] X. Chen and Y. Zhong, "Music electromagnetic imaging with enhanced resolution for small inclusions," Inverse Problems, 25, 015008, 2009.

[4] A. Devaney, "Super-resolution processing of multi-static data using time reversal and music." http://www.ece.neu.edu/faculty/devaney/preprints/ paper02n_00.pdf.

[5] F. Gruber, E. Marengo, and A. Devaney, "Time-reversal imaging with multiple signal classification considering multiple scattering between the targets," J. Acoust. Soc. Am, 115, 3042-3047, 2004. 
[6] F. Cakoni, D. Colton, and P. Monk, The Linear Sampling Method in Inverse Electromagnetic Scattering. Philadelphia: SIAM, 2011.

[7] D. Colton and A. Kirsch, "A simple method for solving inverse scattering problems in the resonance region," Inverse Problems, 12, 383-393, 1996.

[8] G. Bao and P. Li, "Inverse medium scattering for the helmholtz equation at fixed frequency," Inverse Problems, 51, 1621-1641, 2005.

[9] T. Hohage, "On the numerical solution of a three-dimensional inverse medium scattering problem,” Inverse Problems, 17, 1743-1763, 2001.

[10] P. van den Berg, A. van Broekhoven, and A. Abubakar, "Extended contrast source inversion," Inverse Problems, 15, 1325-1344, 1999.

[11] D. Colton, Partial Differential Equations An Introduction. New York: Dover Publications, Inc., 2004. 